\title{
ANTON GRAUVOGL
}

\section{Das Leben ist ein Spiel}

\section{Herrn Professor Dr. Dr. H. H. Sambraus zum 65. Geburtstag gewidmet}

\author{
Summary \\ Title of the paper: Life is a game \\ Sexual reproduction causes the independent combination of genetic elements. But not only the physiology of \\ reproduction but also behavioural physiology is familiar with independent combinations; in this case the \\ behavioural elements while playing. \\ An attempt is made to analyse and discover the difference between the playing behaviour of animals versus the \\ playing behaviour of humans during childhood. \\ The mathematical theory of game also is based upon selective choice. \\ The social aspects of play in animal and human collectives is explained as well as the possibilities of \\ therapeutical games.
}

Key Words: meiose, play behaviour, adolescence, game theory, children's play, therapeutic games

\section{Zusammenfassung}

Die geschlechtliche Fortpflanzung bewirkt die freie Kombinierbarkeit in der Vererbung. Aber nicht nur die Reproduktionsphysiologie, sondern auch die Verhaltensphysiologie kennt eine freie Kombinierbarkeit, hier von Verhaltenselementen im Rahmen des Spielverhaltens.

Es wird versucht, das Spielverhalten zu analysieren und Abgrenzungen zu finden zwischen dem Spiel der Tiere und der Menschen im Kindesalter.

Auch die mathematische Spieltheorie beruht auf Wahlmøglichkeiten.

Die sozialen Aspekte des Spiels furr tierliche und menschliche Kollektive sowie die Möglichkeiten von therapeutischen Spielen werden dargelegt.

Schlusselwörter: Meiose, Spielverhalten, Adoleszenz, Spieltheorie, Kinderspiele, therapeutische Spiele

Den Titel dieses Beitrages kann man exakt wörtlich auffassen. Die Natur (deus sive natura, je nach Anschauung) hat sich etwas einfallen lassen, damit sich das Leben weiter entwickelt. Sie kam auf die überaus kostspielige Idee, statt der ungeschlechtlichen, gleichförmigen, sich nicht fort- entwickelnden Vermehrung eine mit einer Reduktionsteilung ausgestatteten geschlechtlichen Fort-Pflanzung zu nicht gleichförmigen Nachkommen einzuführen. Die Freiheit der Kombination der Erbanlagen in der Meiose war der Gewinn dieser großen Investition.

Diese freie Kombinierbarkeit hat den Wesenszug eines Spieles. Daher ist es auch nicht verwunderlich, dass Modelle der Genetik und der Populationsdynamik an die ChaosTheorie angelehnt werden. Allerdings sorgt bei der Züchtung von Lebewesen die natürliche Selektion und auch die künstliche Selektion durch Tierzüchter dafür, dass nur die „guten“ Kombinationen übrigbleiben, so dass bei stark durchgezüchteten Haustier- 
rassen eine bemerkenswerte Uniformität entsteht.

Was für die Fortpflanzung gilt, kann man auch auf die Verhaltensweisen der Lebewesen übertragen, beides sind ja, streng genommen, physiologische Vorgänge. Neben den sehr essentiellen Verhaltensweisen für Ernährung und Sexualität, für Kampf und Flucht etc. gibt es ein besonderes Verhalten, das alle Verhaltensweisen durchspielt, nämlich das Spielverhalten.

\section{Im Spiel zur Adoleszenz}

Das Spielen kann man als ein Verhalten bezeichnen, das nicht zielgerichtet eine augenblickliche Situation des Individuums ändern will. Es will eben nicht auf eine finale Situation der Nahrungsaufnahme oder des Sexualaktes zusteuern, es hat keinen Ernstbezug.

Im folgenden sind die Verhaltensregeln für das Spiel aufgestellt, wobei wir uns etwas auf die Darstellung des Verhaltensforschers EIBL-EIBESFELDT (1987) stützen, der viel über das Spiel gearbeitet hat:

1. Im Spiel beobachten wir angeborene Instinkthandlungen und erlernte Verhaltensweisen, die zu dem Zeitpunkt ihrer Installation noch gar nicht benötigt werden, z.B. Sexualität.

2. Im Spiel auftretende Instinkthandlungen sind von den ihnen vorgesetzten Antriebsmechanismen abgehängt. Die Spiele erfolgen zwanglos, „ohne Leistungsdruck".

3. Permanent kommt es zu der oben erwähnten freien Kombinierbarkeit von Verhaltensweisen oder der Bruchstücke von solchen. Diese Freiheit ist die Basis eines Dialogs mit Objekten.

4. Eine augenfällige Besonderheit ist, dass Spiele ,in Mode“ kommen, eine Zeitlang mit einem Überschwang an Energie durchgefuhrt werden und dann wieder als nicht mehr up-to-date verworfen werden. Einfaches Experiment: Werfen Sie einen leeren Papiersack in die Schweinebucht. Anspruchsvollere Beobachtungen: Das Spiel eines Affen mit einem Stock - nicht zu verwechseln mit dem Werkzeuggebrauch der Hominiden.

5. Sobald mehrere Artgenossen beteiligt sind, kommt es zum ständigen Rollenwechsel: Verfolger und Verfolgte wechseln sich gegenseitig ab. Folglich könnte man dieses Phănomen als den Dialog mit „Subjekten“ bezeichnen.

6. Das Tier lernt für das spätere Leben anwendbare Kunstfertigkeiten im Verhalten, z.B. das Kämpfen oder das Beuteschlagen.

7. Das Tier hat unbedingt einen Drang zu spielen, einen Spieltrieb, den man in einem Atemzug mit dem Neugiertrieb nennen möchte, nämlich einen Mechanismus, der das Tier drängt, neue Situationen aufzusuchen und mit neuen Dingen zu experimentieren.

Mit dem Spiel haben sich aber nicht nur Verhaltensforscher befasst. Auch praktische Nutztierhalter wie Schweinemäster haben darüber viel nachgedacht (in dieser Abhandlung kommt die Spezies Schwein oft vor, weil an diesen Tieren meine eigenen Untersuchungen stattfanden [1994]). SAMBRAUS (1978) bringt in seinem Lehrbuch ein knappes, aber mit großer Informationsdichte versehenes Kapitel zum Spiel der 
Kälber. Auch hinsichtlich Fohlen wurde bei SAMBRAUS gearbeitet.

Während nämlich normalerweise Tiere nur dann tätig werden, wenn ihnen etwas recht Lebensnotwendiges abgeht - Futter, Wasser, Schlaf oder Sexualität, schließt das Spiel gerade solche Bedarfe aus; es erfolgt nur, wenn sich das Tier einigermaßen ,wohl befindet", also z.B. keinen Hunger hat und auch sonstige Alarmsignale, z.B. beim Hausschwein Schmerzen des Körpers, beim Wildschwein zudem natürliche Feinde oder eine Meute Jagdhunde, nicht das Wohlbefinden stören.

Was tun Menschen oder Tiere, die sich wohlbefinden? Wie eben angedeutet, vernünftigerweise gar nichts, weshalb es - exakt naturwissenschaftlich gedacht - gar kein Verhaltensanzeichen für das Wohlsein geben könnte, wenn das nicht das Spiel wäre. Also ist das Beobachten des Spiels eine ganz wichtige Sache für den Tierschutz, der ja in Mitteleuropa einen hohen Stellenwert hat. Wenn man nicht ganz so exakt denkt, kann man das Schlafen und Ruhen auch als Anzeichen geringer Bedürfnisspannungen ansehen. Vor allem aber sind es die beim Schwein so häufigen Stimmfühlungslaute, die gleichzeitig doch signalisieren, dass zumindest nichts sehr wichtiges passiert, allerdings kann man diese bereits als Dialog mit gewissem Spielcharakter bezeichnen.

Wenn zu berichten ist, wie Tiere spielen, macht man am besten eine Einteilung der einzelnen Verhaltensmuster. So gibt es Solitärspiele. Sogar beim Schwein, das ein sehr soziales Lebewesen ist (im Gegensatz zum mehr einzelgängerisch konstruierten Menschen), macht das Spiel der Ferkel u.U. für sich allein mit den vielen Objekten der Umwelt ein großes Aktivitätspensum aus. Hängt man eine Kette in die Bucht, so können Schweine (anfangs) bis zur völligen Erschöpfung daran rütteln, zerren, beißen. In gleicher Weise, nur für den Menschen nicht so deutlich erkennbar, werden die Gitterstäbe, Tröge und Tränken behandelt und sogar der einfache Boden wird durch Schaben mit der Rüsselscheibe bedient.

Neben diesen Objektspielen gibt es aber auch ausgesprochene solitäre Bewegungsspiele, weniger bei Schweinen, aber um so mehr bei Affen, die Purzelbäume schlagen, oder bei Hunden, die ihrem eigenen Schwanz nachlaufen, oder bei Pferden, die auch allein über die Koppel galoppieren. Alle anderen Spiele sind soziale Spiele. Diese beginnen sehr hăufig mit einer Spielaufforderung, dem Initialspiel. In der Regel ist es ein Hochspringen mit allen vier Gliedmaßen, häufig als Einleitung von Laufspielen. Hunde können zu diesem Zweck ihrem Herren oder - was fälschlich als schlimm gedeutet wird - dessen Kind förmlich ins Gesicht springen, ein „Bussi geben“. Menschenkinder machen gerne einen Hops zur Spielanregung.

Kontaktspiele unter den jungen Schweinen sind mit 10 Prozent aller Schweinespiele anzusetzen. Da ist z.B. das Aufreiten oder das spielerische Erfassen des Schwanzes, aber auch das Besaugen und Massieren des Kumpanen. Zwar kann das Schwanzbeißen aus verschiedenen Gründen auch bei Schweinen recht verletzungsträchtig werden, aber deshalb von „Kannibalismus" bei Schweinen zu sprechen, ist ein entsetzlicher Irrtum; kein Schwein hat die Eigenart mancher sog. niederen Lebewesen, Artgenossen als Nahrungsmittel zu verspeisen. Wird das spielerische Erfassen ernst, so handelt es sich um eine Caudophagie, ein Körperenden-Anfressen. Das ist eine Verhaltensstörung, die bei manchen Spezies gar nicht so selten auftritt und doch wohl dem coping gilt.

Kampfspiele (auch 10 Prozent) werden mit offenem Maul durchgefuhrt. Die Beson- 
derheit dieser Spielform ist es, dass sich unvermittelt Verhaltensübergänge zu ernsten Auseinandersetzungen mit gegenseitigen Beschädigungen zeigen können, was den jungen Ethologen völlig konsterniert (weil nicht sein kann, was nicht sein darf).

Laufspiele nehmen bei Schweinen wie bei Menschen einen breiten Raum ein, vielleicht machen sie ein Drittel aller Spiele aus. Es ist gestattet, wie beim Joggen des Menschen, an einen eigenständigen Lustgewinn zu denken (Opioidschübe) - wie übrigens auch beim Tanzen.

Derartige Laufspiele kann man auch gut zwischen verschiedenen Tierarten beobachten: Manchmal muss schließlich der Betriebsleiter eingreifen, wenn Pferde allzu rastlos, aber mit sichtlichem Vergnügen (man sieht es am sog. Spielgesicht) Rinder jagen oder auch umgekehrt.

Aus all dem bisher Dargestellten geht schon hervor, dass das Spiel instinktoiden Charakter hat. Instinkthandlungen (LORENZ, 1943) - der Instinktbegriff wird heute oft als unscharf abgetan, ist aber ein volkstümlicher Begriff - müssen einen oder mehrere Zwecke für seinen Besitzer haben, sonst wären sie von der Evolution meistens, aber nicht immer, längst wegrationalisiert worden.

Erster Zweck ist das neuronale Einfahren von motorischen Mustern für das spätere Leben. Überdies trainieren die Tiere für den Ernst des Lebens die richtigen Verhaltensweisen ein, je nachdem, was später auf sie zukommt. Das geht bis zum Sexualverhalten. Das kleine, drei Wochen alte Eberlein übt vorsichtshalber jetzt schon, wie man später den Sexualrivalen vertreibt und das Weib richtig packt. Beachte: Hinter jeder einzelnen Aussage dieses launigen Satzes stehen umfassende naturwissenschaftliche Untersuchungen.

Zweiter Zweck des Spieles ist das Begreifen der unbelebten Umwelt. Dieses „Begreifen" muss doppelsinnig verstanden werden; nämlich motorisch und zentralnervös bis zur Neocortex. Nur so kann das Tier zu einem systematischen Erkunden der Umwelt kommen.

Wirft man einen Ball in den Käfig, wird er ähnlich wie ein Kumpan behandelt. Menschenkinder und die Kinder unserer großen Säuger „begreifen“ ihre Umwelt. Unser Säugling macht das mit den Fingerchen, das Ferkel mit dem Mäulchen (weil es auf den Vorderfüßen ja stehen muss). Die Rüsselscheibe ist deshalb mit derselben Anzahl Nerven ausgestattet wie die Finger des Menschen. Überhaupt können Schweinekinder ebenso denken wie Menschenkinder etwa bis zu zwei Jahren. Und prägungsähnliche, das spätere Empfinden formende Erlebnisse spielen hier wie dort eine große Rolle.

Dritter Zweck ist der Aufbau von sozialen Beziehungen des Tieres zu seinen Kumpanen. Cum panes $=$ Tiere, die in einer Umfriedung gemeinsam ihr Brot essen, sind für das Beobachten von sozialen Verhaltensregeln eine ganz wichtige Einheit, weil die Nahrungsaufnahme doch einen großen Teil der gesamten Motorik in Anspruch nimmt. Auch Sexualität und Aggression sind in einer Buchtengemeinschaft von großer Bedeutung. Beim Spielen sieht das dann im Ferkelkäfig so aus: Ferkel springen gegenseitig auf (Sexualverhalten), verstecken sich (Fluchtverhalten), zeigen Teile des Rivalenkampfes, probieren das Wühlen und das Rütteln und spielen exakt wie Menschenkinder Fang-mich. Man ist ganz merkwürdig berührt, wenn man abwechselnd Kindern und Ferkeln zusieht, wie völlig gleich dabei das Verhalten ist. Es ist erstaunlich, wie 
die Rollen laufend vertauscht werden und z.B. Verfolgte und Verfolger wechseln, wenn dieser zum Anstupsen kommt. Die Verhaltensforscher sind der Ansicht, dass auf diese Weise die Tierkinder in einen Dialog mit den Artgenossen eintreten.

Neben der Aufzählung der drei Zwecke wollen wir schon unter dieser Ziffer II eine Eigenart des Spieles erwähnen, die wir allen drei kommenden Ziffern gemeinsam voraussetzen müssen:

Das Spiel ist lustbetont. Das ist zwar nicht neu, dass dem Menschen und den Tieren das Spielen „Spaß macht“. Aber es gibt doch recht exakte Untersuchungen an Tieren hierzu. Besonders bekannt sind experimentelle Arbeiten geworden, die eine Belohnungsdressur damit durchführen können, dass Affen zur Belohnung einer Leistung schlicht aus dem Fenster schauen dürfen. Es ist also nicht nur die Bewegungsrhythmik, die beim Ringelreihen den Kindern oder beim Fußballspiel der Erwachsenen körpereigene Morphine (o.g. Opioide) freisetzen mögen, sondern doch wohl ein eigenständiges instinktoides Spielverhalten, das zu einem fakultativ erlernten Verhalten führen kann.

Instinkte haben eine angeborene Motivation, einen Soll-Wert im genetischen Programm., der laufend mit dem Ist-Wert des Organismus verglichen wird. (Früher hat man vom Stauphänomen gesprochen, z.B. von LORENZ' psychohydraulischem Modell 2 (1978)). Also benötigt das Lebewesen mehr oder weniger ein bestimmtes Quantum Spiel. Vom Spiel ist so ein marginales Quantum eher weniger notwendig im Verhältnis zu Angst und Aggression etc. Man sagt, die Spielmotivation hat einen geringen Durchlasswert vor den Pforten des Großhirns zu neuen Taten. - Demgegenüber haben Kampf und Brutverteidigung etc. einen hohen Durchlasswert; das bedeutet, diese Antriebsmechanismen haben den absoluten Vortritt in der Verhaltenssteuerung: Versuchen Sie einmal einen Frischling einer führenden Bache zu streicheln; die fährt auf Sie zu wie ein überschweres Motorrad. Es wäre ja auch äußerst fatal für ein Ferkel, wenn die Brutverteidigung bei der Bache nicht sofort einklinkt.

Generelle Regel: Das Einüben von Bewegungsmotoriken oder das Training des schnellen, richtigen Reagierens auf Reizsignale von Objekten oder lebenden Artgenossen oder Nichtartgenossen sind zeitaufwendige Exerzitien. Für solche bedarf es einen störungsfreien, entspannten Raum. Hat das jugendliche Tier keinen solchen Freiraum, bekommt es keine Übung und ist „im freien Spiel der Kräfte“ des Daseinskampfes doch etwas benachteiligt.

\section{Hintergründige Spieltheorien}

Es gibt eine eigene wissenschaftliche Lehre, nämlich von den Spieltheorien. In dieser Lehre werden die Vorteile und die Nachteile, in unserem Fall eines bestimmten Verhaltens, mathematisch durchkalkuliert, wobei schlussendlich im natürlichen Selektionsdruck das Individuum mit dem günstigsten Verhalten rein rechnerisch übrig bleibt. Wir wollen dies an einem extrem einfachen amerikanischen Beispiel, das im IMMELMANN et al. (1988) vorgetragen wird, verdeutlichen:

Das sog. Chickenspiel (Feiglingsspiel) besteht darin, dass zwei mutige Fahrer vor einer Tribüne voll Zuschauern in (alten) Fahrzeugen aufeinander zu fahren. Wer als erster die Nerven verliert und vom Kurs abweicht, hat verloren. Der andere Fahrer heimst den von den Zuschauern gestifteten oder gewetteten Gewinn ein (200 Dollar). 
Weichen beide aus, kriegt jeder die Hälfte des Gewinnes. Das gilt auch für die Fälle, wo ab einem ,point of no return" keiner ausweicht und es sicher zu einem Unfall kommt (eine Havarie soll pro Auto 1000 Dollar kosten).

Nun kann man eine Rechnung aufmachen, was mehr bringt: Wenn beide ängstlich sind, öfter ausweichen und deshalb die Fahrzeuge mehr heil halten. Oder wenn einer mutig ist und dann auch viel Geld einheimst, aber öfter eine Havarie hat. Oder wenn einer ängstlich ist .... . Im Hintergrund steht das Problem des ,point of no return“! (Für Insider: In Büchern finden sie ein ebenfalls noch mathematisch leicht überschaubares Beispiel, das "Gefangenendilemma“.) Solche Rechnungen kann man z.B. auch sehr gut über das Zusammenleben von Tiergesellschaften aufstellen. Bekannt geworden ist die Modellrechnung über Tauben und Falken. Es ist nämlich keineswegs so, dass die Falken alle Tauben vollständig aus einem gemeinsamen Lebensraum verdrängen, wie das ein Student mit etwas schlichtem Verstand vermuten könnte, abgesehen davon, dass auch eine Rolle spielt, wer zuerst da war. Am Schluss der Rechnung kommt man zu evolutionsstabilen Strategien (ESS).

Man kann viele Probleme, welche die belebte oder unbelebte Umwelt stellt, durchspielen. Das macht auch die Natur und kommt schließlich immer wieder zu einem Art Fortschreiten im Rahmen vieler Regelkreise: die „Evolution“ nennen wir Menschen dieses Geschehen und finden auch hier wieder eine Bestätigung der These unseres Titels, dass das Leben ein Spiel ist.

IV.

Das Spiel der anima cognitiva

Das Spiel der Tiere wurde im vorletzten Kapitel dargestellt und es wurden einige Besonderheiten gefunden, die uns lebhaft an das Verhalten von Menschenkindern erinnerten. Generell meinen die Psychologen, dass der Mensch sein Leben lang ein Spieler bleibt. LORENZ (1978) formulierte, dass beim Menschen die Neugier ein persistierendes Jugendmerkmal sei, was ungefähr dasselbe bedeutet.

Jedoch, die Übergänge sind gleitend. Besonders eindrucksvoll wird von den Spielen völlig erwachsener Delphine berichtet. Weniger bekannt ist, dass man auch einiges bei Nutztieren sehen kann: Stiere und Eber spielen gerne mit den Kleinen, jedenfalls mehr als die Mütter (denen vielleicht die Brutfürsorge weniger Zeit lässt). Bei Pferden sieht man, dass innerhalb einer Herde eine Art Tante das junge Volk beaufsichtigt und sich gelegentlich trotz ihres massigen Körpers in die Laufspiele einklinkt. Wir wissen, dass Spiele allgemein ansteckend sind; ich finde aber, dass dies bei Menschen und Großsäugern besonders anschaulich ist.

Interessant sind exakte Untersuchungen, ob die geschlechtsspezifischen Unterschiede im Spiel der Menschenkinder allgemein verbreitet sind und auch bei den Säugern bestehen. EIBL-EIBESFELDT (1995) hat beim sehr urtümlichen Stamm der Himba Spiele, das Stein- und Speerwerfen, ausgezählt und ein Verhältnis von 272 zu 93 zwischen Maskulinum und Femininum gefunden. Nach meinen Beobachtungen sind bei Ferkeln männliche Tiere viel aggressiver beim Spiel als weibliche. Das gilt wohl auch für Menschen und das schon im Lebensalter von zwei Jahren, so wird berichtet. SAMBRAUS (1978) stellt bezüglich Sexualität fest, dass beim spielerischen Aufsprung fünfmal soviel Stierkălber wie Kuhkälber tătig werden. 
Es gibt aber auch eine Verhaltensweise, die man beim Menschen in gleicher Weise wie bei Säugern nur in der Adoleszens findet als besonderes Neugierverhalten. Jugendliche Menschen und Tiere besitzen eine spezielle Lebensphase, in der sie dem vertrauten Lebensraum zu entrinnen suchen. Dieser Wandertrieb zeigt starke Bezüge zum Erkundungsverhalten (dem aktiven sensorischen Untersuchen der Umwelt). Bei unseren heutigen Haustierrassen ist der Antrieb, der offensichtlich auch dazu dienen sollte, der Spezies neue Lebensräume zu erschließen und Inzucht zu vermeiden, so weit reduziert, dass dem Tierwirt bei der Umfriedung von Weiden und Buchten für jugendliche Tiere keine sehr erheblichen Sorgen entstehen.

Im wesentlichen sind es aber doch sieben Sachverhalte, die das Spiel der Tiere und der Menschen unterschiedlich erscheinen lassen:

1. Menschenkinder lassen nach dem zweiten Lebensjahr ein Ich-Bewusstsein erkennen. Z.B. erkennen sie sich selbst im Spiegelbild und versuchen einen Farbfleck richtigerweise von ihrem Kopf und nicht vom Spiegel wegzuwischen. Das kennt man sonst allenfalls von Schimpansen und vielleicht noch von zwei bis drei anderen Primaten. Dieses Selbsterkennen hat innerhalb des Sozialverbandes Vorteile.

2. Der wichtigste Vorteil ist der Wechsel von Selbstbezug zum Partnerbezug. Was können jetzt herrliche Rollen gespielt werden! Das beginnt mit intensivem Geben und Nehmen von (persönlichen) Spielzeugen an Spielpartner und geht weiter zu Spielen mit sozialen Rollen: Der früher so bekannte Kinderkaufladen mit Verkäufer und Kunden, das war eine wertvolle kognitive Bereicherung. Rollenspiele finden in der Puppenküche statt. Schließlich kommt eine Phase unweigerlich mit Onkel-Doktor-Spielen, vielleicht mit einem Aufblitzen von Sexualität, immer aber mit Ansätzen zur Empathie für den Partner, zum „Hineinversetzen" in das Du. Schon allein das Ausloten, wie weit kann das Ich ungestraft seine eigenen Aggressionen beim Partner ausspielen, ist eine zentrale Maßnahme zur Eingliederung in den Sozialverband.

3. Eine Eigenart der Spiele auch bei Tieren ist eine gewissen Unermüdlichkeit von Wiederholungen. Man kann annehmen, dass für Menschenkinder neben dem zuverlässigen Einfahren von motorischen Mustern durch die Repetitionen das Fokussieren der eigentlich neuen Erkundung und Erkenntnis erreicht wird. Beim Drücken auf den Hebel senkt sich die Ladefläche des Spiel-Lkw ab; ist das Zufall? ist es eine Koinzidens mit einer anderen Motorik? Wie stark in welche Richtung muss der Hebel gedrückt werden, um gerade noch den Erfolg zu erzielen? Mit unermüdlichem Probieren kommt das Kind zur instrumentalen Exploration (z.B. mit Stöcken), zu Konstruktionsspielen (einen Turm aus Klötzen bauen) und zu neuen Lösungen eines Problems, z.B. über eine Bank die geliebten Klaviertasten zu erreichen.

4. Erstaunen erwecken die Kinder bei uns, wenn sie mit fiktiven Objekten und Ersatzobjekten spielen. Bereits zweijährige Kinder sind in der Lage, mit einem Stück Holz als Ersatz eines Kleinstkindes zu spielen. Und endlich werden an fiktiven Objekten fiktive Handlungen, z.B. Stillen oder Füttern, durchgeführt. 
5. Menschen und Tiere benutzen Lautäußerungen, um Informationen an Artgenossen (intraspezifisch) oder Nicht-Artgenossen (interspezifisch) weiterzugeben. Man kommt durch größere Studien auf etwa zwei Dutzend (unscharfer Zahlenbegriff absichtlich) lautliche Äußerungen jeweils bei Rindern und Schweinen, was für Tiere sehr viel ist. Bei den Menschen sind die Verhältnisse ganz anders. Schon im Vorschulalter sind die Kinder zu ausgedehnten Sprachspielen in der Lage, beginnend mit Text auf Ringelreihen. Die heranreifenden Hirnfunktionen erlauben uns, an Kinder nicht nur Mitteilungen über die präsente Situation, sondern auch solche aus der Vergangenheit (Märchen) mit reichlicher, oft spielerischer Phantasie heranzutragen. Schließlich spricht der Mensch über Sachverhalte, die gar nicht gegenständlich sind, z.B. Kultur und Moral. Durch seine unvorstellbaren geistigen Fähigkeiten (wir rechnen mit hundert Milliarden Neuronen) wird die Sprache in ihrer Kombinationsfähigkeit zum gewaltigen Sprung der Evolution.

6. Nur das (Menschen)Kind ist fähig, im spielerischen Malen bereits mit gestaltgebenden Zeichnungen und Malereien zu arbeiten. Malprodukte der Affen sind oft recht schön, aber sie sind keine gegenständliche, bildende Kunst wie beim Kind. Gerade kindliche Zeichnungen in ihrer Reduktionsfähigkeit verblüffen uns: die Kopf-Füße-Männchen oder die Häuser aus einer Handvoll Strichen. Beobachten wir anschließend die Bildschrift der alten Sumerer und schlussendlich die Schriften von heute als alles übertreffendes Kulturgut, so erkennen wir den ungeheuren Kulturschub, den der Mensch in wenigen tausend Jahren leistete.

7. ARISTOTELES, dieser unglaublich hellsichtige Hellene, erkannte bereits, dass der Traum Wesenszüge des Spiels beinhaltet. Träume hat zwar auch der Hund, der im Schlaf Beute jagen zu scheint. Die Fähigkeit des Menschen, mit Wahrnehmungen im Traum Situationen durchzuspielen, dürfte aber einzig sein. Der Traum unterscheidet sich vom Werk des Tages dadurch, dass seine Werke nur Spiele mit Möglichkeiten sind, meint Aristoteles.

Im Traum bauen wir Luftschlösser. Schlimm ist es, wenn sich die Grenzen zwischen Traum und Wirklichkeit verwischen. Durch den Realitätsverlust wird der Mensch in seinem sozialen Verhalten unberechenbar. Diktatoren aller Zeiten neigten dazu. „Ich bin ein Hasardspieler“, sagte Hitler (authentisch!) und beschwor die größte Katastrophe in der Geschichte der Menschheit herauf.

Das Durch-Spielen verbindet den Traum mit den höchsten Leistungen des Großhirns, nämlich Gedächtnisinhalte für kognitive Handlungen frei zu kombinieren. „Als sich in der biologischen Menschwerdung der Verstand entfaltete", meint HASSENSTEIN (1992) - und man muss ihn ob seiner knappen Sprache wörtlich zitieren - ,scheint der produktive Teil der Intelligenz, der intellektuelle Variations- und Suchmechanismus, seine Funktion von der Spielsteuerung entlehnt zu haben, wo ein entsprechendes Abwechseln und Variieren bereits perfekt vorgebildet war". 


\section{Spiel zur Befriedung der Massen}

„Panem et circenses“, schrie das römische Volk - und der Pöbel wohl am lautesten zur Kaiserloge hinauf. Es ist eben falsch, dass neben Angst und Aggression nur Hunger und Sexualität die kardinalen Antriebe des Menschenverhaltens sind. Das Sexualverhalten löst nur das Problem, mittels wohldosierter Mischung von Angst und Aggression im richtigen Augenblick anzudocken, wie die Sojus an die Mir, um etwas hinüber zu bringen. Aber Spiele bedeuten Freiheit. Spiele - heute sind es Spielfilme sind das Surrogat für eigene, oft so geheimgehaltene, nie erfüllte Träume.

Prunkprozessionen als Schauspiel für das Volk kannten schon die alten Ägypter. Aber richtig spannend wurde es, ,action" kam in die Sache, als man Mensch und Tiere gegeneinander kämpfen ließ in den Kampfspielen, die im alten Rom allgegenwärtig waren. 50.000 Menschen lockten sie - auf einmal - in das Kolosseum, die Tausende und Abertausende von möglichst großen und wilden Tiere sterben zu sehen wünschten (GRAUVOGL, 1984). Zum größeren Ruhm des Kaisers, auf den circenses eines einzigen Festes. Für den Kaiser stand klar die Erhaltung seiner Macht im Vordergrund. Aus diesem Grund finanzierte er auch die sehr teuren Spiele, die seine Staatskasse arg belasteten. Die Spiele hatten einmal die Aufgabe, den Kaiserkult zu fördern. Dies wurde erreicht durch vielerlei Einlagen, sowie durch die Veranstaltung an sich, die stets kostenlos und mit einer Gratisverköstigung verbunden war. Zum zweiten garantierten die Spiele dem Kaiser in gewissem Umfang Schutz vor sozialen Unruhen. Das ganze arbeitslose Proletariat von Rom, das einen latenten Unruheherd darstellte, war so wenigstens zeitweilig von der Straße weg und fand im Amphitheater ein Aggressionsventil für den ,angestauten“ Ärger.

Der Ausweichcharakter des Spieles gegenüber stark unlustgetönter Situationen ist in seinem Therapiewert nicht zu unterschätzen. Das wissen die Menschen. Die Vergnügungsindustrie, die der Zerstreuung für den von Ängsten geplagten Menschen dient, ist ein ungeheuerer Wirtschaftsfaktor geworden. Das fängt an bei der Spielkultur für Kinder (Jagdspiele mit Würfel) über Monopoly, bis zu Brettspielen für Erwachsene in allen Kulturkreisen, und endet bei Video und Computerspielen.

Man sollte die großen Karnevalsveranstaltungen fast als therapeutisch wertvolle Ereignisse bezeichnen; kann hier doch der schlichte Bürger seine Sorgen vergessen, indem er in die Rolle eines anderen, heiteren Menschen schlüpft.

Heiterkeit und Frohsinn begleiten die Spiele des gesunden Erwachsenen. Ist er krank, insbesondere psychisch, fehlt ihm der Humor. Aber den kann man ihm durch therapeutisches Spielen wieder beibringen.

Nun ist bekannt, dass es durch die Angebote der multimedialen Unterhaltung zu einer Überflutung mit Reizen kommt, welche die ohnedies in hoher Populationsdichte lebenden und deshalb schon gereizten Menschen zusätzlich belastet. Dem homo cupidus rerum novarum (deutsch richtig: neuerungssüchtigen Menschen) bringt die Gier nach Neuem in schwere Stresssituationen mit entsprechender Auswirkung auf Kreislauf, Hormonhaushalt und Immunstatus (Suppression). Mit Sedativa und Schlafmitteln ist hier nur sehr vordergründig geholfen. Die eigentliche Therapie besteht in einem Bewusstwerden der hintergründigen Zusammenhänge der Reizüberflutung, so dass ein bewusstes Gegensteuern möglich wird: Durch Spielen mit einfachem Gerät. 
Denken wir an die Verantwortung für unsere Kinder. Ständig aktivierte Bereitschaft, auf Reizsignale der großen Antriebe Angst, Aggression, und in anderen Teilen der Welt auf Hunger zu antworten, erzeugt Störungen des Spielverhaltens. Gestörtes Spielverhalten bedeutet aber gestörte Verhaltensentwicklung im Ganzen.

Findet man bei Menschen meistens eine Umwelt mit zuviel Reizen, sehen wir die Problematik der modernen Nutztierhaltung in großen, intensiven Tierhaltungen, umgekehrt in der Reizverarmung. Während unsere Menschenkinder in den Städten wegen zu hoher Reizanflutung Stresserscheinungen zeigen, erregt sich manches Schwein außerordentlich, weil es, trotz aller Reizschwellensenkung zu keinem, seinem genetischen Programm adäquaten auslösenden Reiz finden kann.

Zur Prophylaxe und zur Therapie der Reizschwellensenkung gibt man heute schon Strohbüschel, Ketten und Kisten in die Schweinebuchten als Spielzeug. Und es werden sogar zu Gunsten des enrichment Bedenken der hygienischen Nachteile hintangesetzt. Wir werden noch zu mancher Umwertung der Qualitäten von Tierschutzmaßnahmen kommen. Viele meinen heute noch, dass eine Schweinebucht möglichst groß und sauber sein und einen weichen Boden besitzen soll. So dachten die Betreiber von Menschenaffen-Stationen auch noch bis vor zehn Jahren. Heute sieht es in den Affenhäusern aus, als wenn Menschenkinder randaliert hätten. Verbeulte Plastikeimer, zerrissene Papiersäcke und anderes mehr liegen auf einem Fliesenboden, den man wenigstens leicht reinigen kann; in womöglich engen Nestern, weil Kinder ganz gern in Tuchfühlung leben.

Zum Abbau von Stressrelikten und zur Bremsung von psychischen Störungen u.a. empfiehlt BENESCH (1995) - ein hochverdienter Forscher über Spieltherapien - den Einsatz von elektronischem Spielgerät. Das ist eine ganz bemerkenswerte Sache. Denn allzu oft werden derartige Geräte generell abgelehnt - in Assoziation an die ,Spielhöllen" der Großstadtjugend oder gar an süchtig machende einarmige Banditen, die wir aus Westernstorys kennen.

Derselbe Forscher kennt eine positive Wirkung des Spiels, die er Verstärkung nennt. Und wiederum ziehen wir ein wörtliches Zitat vor, um eigene, wahrscheinlich weitschweifig werdende Überlegungen zu vermeiden. „Leistung braucht Bestätigung. Der Spieler erlernt Gewinnen und Verlieren, aber er lernt auch beide in ihrer Bedeutung nicht zu überschätzen. Dadurch verbessert er seinen Erfolgs-Misserfolgshaushalt im alltäglichen Leben".

Je kleiner der Mensch das Glück des Erfolgs einschätzt, desto beständiger glücklich wird er sein. Man kann formulieren, in Erinnerung an Arbeiten von FREUD zur übergroßen Fixierung auch des Libido-Besatzes an ein Objekt (oder an eine Person): Gebt das Glück in kleinen Groschen aus, z.B. im Kartenspiel.

Systemanalytisch gedacht muss es Bezüge des Spieltriebes zu den Fürsten in der Hierarchie der Instinkte geben, zu Angst und Aggression.

Richtig kennen wir das Spiel mit den kleinen Ängsten. Viele Gesellschaftsspiele besitzen das animierende Bestandteil „Risiko“. Die erregende Situation eines Abenteuers, das es zu bestehen gilt, ist nicht nur von Abenteuerspielplätzen bekannt. Es zieht sich eine glatte Linie weiter über Geisterbahnen, Trekking, bis zu Überlebenskursen. „Wir sind noch einmal davongekommen", das fordert die pure Lust am Leben ungeheuer. 
Ich meine, dass dies auch die Wurzel für das Erleben der „Angstlust“ in Gruselfilmen sein mag. Selbst bei größeren Tieren machen wir die Beobachtung, dass nach einem überstandenen leichten Schrecken das Spiel erst richtig losgeht.

Die letzte große Gruppe an Spielen, die gesondert abzuhandeln sind, betreffen die Kampfspiele. Problemstellung für die Evolution war wahrscheinlich, wie Kämpfe mit u.U. verheerenden Folgen unter Artgenossen vermieden werden können. Wohl gibt es hierzu ein großes Bukett von Signalen im Konkurrenzkampf, aber das wirkt nicht eigentlich erregungsableitend. So kam es zu dem weiten Bereich von Kampfspielen, sportlichen Wettkämpfen bis hin zum ritualisierten Kampf, z.B. in besonderen Schautänzen.

Diese spannungsabführende „Ventilsitte“ (EIBL-EIBESFELDT, 1995) in Kampf- und Wetteiferspielen verlangt nicht unbedingt die persönliche Teilnahme der Bezugspersonen: „Das Publikum geht voll mit“ in den großen Arenen der Fußballspiele, sagt der Reporter. Der Zuschauer fährt nach Hause mit der Befriedigung, an einem großen Kampf teilgenommen zu haben, und das in der schutzgewährenden Gemeinschaft einer Partei für das eine oder andere Kampfkollektiv. Für diese geistige Masturbation heizt sich die große Masse der Zuschauer emotional zu einem „Hexenkessel“ mit höchsten Amplituden an Lärm auf. Und Lärmen ist allemal ein wesentlicher Bestandteil vom Spielen!

Ich denke zweitausend Jahre zurück an die Kampfarenen im alten Rom. Dort beobachtete man bei den Gladiatorenkämpfen allerdings kulturelle Reduktionen, die keine positive Fortentwicklung der römischen Population anzeigten.

\section{Schlussbemerkung}

Was ist als Schlussbemerkung vorzutragen? Diesseits aller Evolutionstheorie ist auf Erden eine freie Kombinierbarkeit von Bausteinen vorgesehen. Die Segnungen der Freiheit finden sich als Spiele auch in den Bereichen des Verhaltens vor: In Aufzucht, Kindheit und Erwachsenenalter bewirken Spiele eine Erweiterung der reaktiven Möglichkeiten für den Einzelnen und eine Festigung der sozialen Gefüge zum Nutzen des eigenen Erbguts.

Ich schließe mit der vierten Strophe eines unterlegten Textes von CLAUS LUDWIG LAUE für ein Old Scottish Folk:

Nehmt Abschied, Brüder, schließt den Kreis, das Leben ist ein Spiel; und wer es recht zu spielen weiß, gelangt ans große Ziel.

\section{Literatur}

ARISTOTELES:

De anima. Georg Picht Vorlesungen und Schriften. Klett-Cotta Stuttgart. 2. Auflage 1992 BENESCH, H.:

Enzyklopădisches Worterbuch - Klinische Psychologie und Psychotherapie. Psychologie Verlags Union 1995 
EIBL-EIBESFELDT, J.:

Grundriss der vergleichenden Verhaltensforschung. Piper München. 7. Auflage 1987

EIBL-EIBESFELDT, J.:

FREUD, $\mathrm{S}$ :

Die Biologie des menschlichen Verhaltens. Piper München. 3. Auflage 1995

Abriss der Psychoanalyse. Fischer Taschenbuch Verlag Frankfurt am Main 1972

GRAUVOGL, A.:

Das Verhaltnis des Menschen zum Tier im alten Rom am Beispiel der Tierhetzen. Bayer. Landw. Jahrbuch 61 (1984), 985 - 994

GRAUVOGL, A.:

Schweine brauchen Brot und Spiele. Schweinezucht u. Schweinemast 41 (1994) 2, 30 - 32

HASSENSTEIN, B.:

Klugheit. Zur Natur unserer geistigen Fähigkeiten. Serie Piper Band 1109, München 1992

IMMELMANN, K.; SCHERER, K.R.,; VOGEL, Chr.; SCHMOOCK, P.:

LAUE, K. L.:

(Hrsg.): Psychobiologie - Grundlagen des Verhaltens. Gustav Fischer Verlag, Stuttgart 1988

LORENZ, K:

LORENZ, K.:

Nehmt Abschied Brüder. In: Laute, schlag an. St. Georg Verlag Augsburg 1952

Die angeborenen Formen morglicher Erfahrung. Z. Tierpsychol. 5 (1943), 235 - 409

Vergleichende Verhaltensforschung. Springer Wien/New York 1978

SAMBRAUS, $\mathrm{H}$.-H.:

(Hrsg.), Nutztierethologie. Paul Parey, Berlin und Hamburg 1978

Eingegangen: 30.05 .2000

Akzeptiert: 05.07.2000

Anschrift des Verfassers

Prof. Dr. ANTON GRAUVOGL

Fasanenweg 12a

D-85661 Forstinning 